\title{
Conocimientos, actitudes y prácticas de niñas y niños sobre educación sexual integral en ocho municipios de Colombia
}

\section{Knowledge, attitudes and practices of girls and boys on comprehensive sexual education in eight municipalities of Colombia}

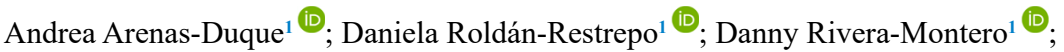 \\ Sandra-Marcela Sánchez-Molano ${ }^{1}$; ${ }^{\mathbb{D}}$ Juan-Carlos Rivillas-García ${ }^{1}$ (D)
}

Forma de citar: Arenas-Duque A, Roldán-Restrepo D, Rivera-Montero D, Sánchez-Molano SM, Rivillas-García JC. Conocimientos, actitudes y prácticas de niñas y niños sobre educación sexual integral en ocho municipios de Colombia. Salud UIS. 53: e21016. doi: https://doi.org/10.18273/saluduis.53.e:21016 (c) (1)

\section{Resumen}

Introducción: la educación integral para la sexualidad permite dotar a niñas/os y adolescentes de conocimientos basados en datos empíricos, habilidades, actitudes y valores que fortalecen sus procesos de empoderamiento para disfrutar del derecho a la salud, el bienestar y la dignidad humana; además, les permite comprender cómo proteger y velar por la garantía de sus derechos a lo largo de su vida. Objetivo: describir los conocimientos, actitudes y prácticas en educación sexual integral de 6645 niñas y niños escolarizados en ocho municipios priorizados de Colombia. Metodología: Ocho municipios fueron seleccionados según indicadores socio-demográficos, embarazo adolescente y violencia de género. Los criterios de inclusión fueron definidos: niñas/os escolarizados de entre nueve y 11 años de edad y con residencia habitual en el municipio. Una encuesta de Conocimientos, Actitudes y Prácticas fue diseñada, validada y aplicada para recolectar datos de 5600 hogares, 50 instituciones educativas y 6645 niñas/ os. Se realizó un análisis descriptivo de la muestra, profundizando en los datos obtenidos del cuestionario aplicado a niñas/os. Resultados: se encontraron altos porcentajes de desacuerdo de violencia directa contra la mujer y validación de formas sutiles de violencia en las niñas/os. Los niños reciben más castigos físicos que las niñas. El término sexualidad es desconocido por la mayoría de niñas y niños, quienes creen conocerlo lo dialogan principalmente con su mamá y más del $20 \%$ no lo hablan con nadie. Las niñas y niños participantes están iniciando relaciones sexuales a edades tempranas sin previa educación en sexualidad. Conclusiones: las niñas y niños interiorizan y reafirman roles tradicionales de género y tienen percepciones erradas sobre la sexualidad, por lo cual, es necesario profundizar en el significado de sexualidad y sobre la violencia basada en género. Esto con el fin de desarrollar capacidades en la toma de decisiones, que influyan positivamente en sus proyectos de vida.

Palabras clave: Educación Sexual; Sexualidad; Niñas; Niños; Hogares; Instituciones Académicas.

1. Asociación Profamilia. Bogotá, Colombia.

Correspondencia: Andrea Arenas-Duque. Dirección: Calle 34 14-52 (Bogotá) - Teléfono: +57 3175049728. Correo electrónico: andrea.ad131@gmail.com 


\begin{abstract}
Introduction: Comprehensive sexuality education enables children and adolescents to be provided with knowledge based on empirical data, skills, attitudes, and values that strengthen their empowerment processes to enjoy their right to health, well-being, and human dignity. Additionally, it allows them to understand how to protect and guarantee their rights throughout their lives. Objective: To describe the knowledge, attitudes, and practices in comprehensive sexuality education of 6,645 school age children enrolled in eight prioritized municipalities in Colombia. Methods: Eight municipalities were selected according to its sociodemographic characteristics, and indicators of teenage pregnancy and gender-based violence. The inclusion criteria were: children going to school between nine and eleven years old and habitually residing in the municipality. A Knowledge, Attitudes and Practices survey was designed, validated and applied to 5,600 families, 50 schools and 6,645 children. A descriptive analysis of the sample obtained was carried out, delving into the data obtained from the children's survey. Results: High percentages of disagreement regarding direct violence against women were found in children's responses, as well as validation of subtle forms of violence. Boys received more physical punishment than girls. The term sexuality is unknown by the majority of surveyed boys and girls, those who believe to understand it have discussed it mainly with their mothers and more than $20 \%$ have not spoken about it with anyone. The participating boys and girls are initiating sexual relations at an early age without prior sexuality education. Conclusions: Girls and boys internalize and reaffirm traditional gender roles and have wrong perceptions about sexuality. That is why, to develop with them decision-making skills that will have positively influence in their life projects it is necessary to deepen the meaning of sexuality and genderbased violence.
\end{abstract}

Keywords: Sex Education; Sexuality; Boys; Girls; Homes; Schools.

\section{Introducción}

La ausencia de educación integral para la sexualidad desde tempranas edades puede socavar el logro de la agenda de desarrollo sostenible por sus efectos a nivel individual, social y político; investigaciones previas reportan que 750 millones de mujeres y niñas se casaron antes de los 18 años ${ }^{1,2} .16$ millones de embarazos adolescentes (15 a 19 años) representan un $11 \%$ de los nacimientos en el mundo, aproximadamente un millón entre niñas menores de 15 años $^{3}$. Los determinantes que explican el embarazo adolescente se relacionan con las desigualdades sociales, el matrimonio forzado a corta edad, la violencia de género (VBG), la pobreza y la falta de oportunidades de realización personal como estudiar $^{4}$. Necesidades insatisfechas en salud sexual y reproductiva (SSR) están resultando en alrededor de tres millones de abortos inseguros en mujeres de 15 a 19 años $^{3}$. Sumado a la necesidades insatisfechas, los determinantes sociales y los estereotipos de género dan oportunidades para las formas de VBG que explican las relaciones de poder y los embarazos no deseados en adolescentes ${ }^{4,5}$.

En 2015, la educación integral para la sexualidad ocupó un lugar fundamental en los Objetivos de Desarrollo Sostenible, en los que se resalta garantizar el acceso universal a los servicios de SSR, incluidos los de planificación familiar, información y educación integral para la sexualidad en las estrategias y los programas nacionales ${ }^{2}$. La educación integral para la sexualidad permite dotar a niñas/os y adolescentes de conocimientos basados en datos empíricos, habilidades, actitudes y valores que fortalecen sus procesos de empoderamiento para disfrutar del derecho a la salud, el bienestar y la dignidad humana; además, les permite comprender cómo proteger y velar por la garantía de sus derechos ${ }^{6}$.

En Colombia, una de cada seis mujeres se convierte en madre antes de cumplir 19 años ${ }^{7}$. Aunque se ha avanzado en la reducción de las tasas de embarazo de mujeres de 15 a 19 años, esta reducción no se refleja en niñas de 10 a 14 años, quienes se enfrentan a un mayor riesgo de embarazo temprano y son las principales víctimas de violencia sexual ${ }^{8}$. A partir de la revisión de los registros de estadísticas vitales, el Ministerio de Salud alertó sobre el riesgo de incremento de los nacimientos en adolescentes entre los 10 a 14 años? .

El $14 \%$ de las mujeres en Colombia tuvo su primera relación sexual antes de los 15 años y este porcentaje llega al $30 \%$ entre los hombres ${ }^{5}$. Cuando el inicio de las relaciones sexuales es antes de los 14 años, es tipificado como violencia sexual ${ }^{10}$. Estos indicadores varían mucho entre los contextos urbanos y rurales; zonas afectadas por el conflicto armado y la pobreza ${ }^{8}$. Según la Encuesta Nacional de Demografía y Salud (ENDS) 
2015, 9 de cada 10 niñas que quedan embarazadas en Colombia abandonan la escuela. De allí que se hable en numerosos casos del embarazo en la adolescencia como un círculo que impide que se cierren las brechas de desigualdad.

De acuerdo con el Departamento Administrativo Nacional de Estadística, Uribia, Dibulla y Aracataca son los municipios más poblados y donde hay el mayor número de niñas/os menores de 18 años. Bahía Solano y Caloto, presentan el menor número de personas en este grupo poblacional. En cuanto a la distribución entre la población urbana, rural y dispersa, los municipios de Dibulla, Uribia y Caloto, presentan más personas que viven en el área rural. Por otra parte, 13 millones de colombianos se encontraban en condición de pobreza monetaria en el año 2018 siendo Chocó (61,1\%), La Guajira (53,7\%), Cauca $(50,5 \%)$ y Magdalena $(46,6 \%)$ los departamentos con mayor porcentaje de población pobre ${ }^{11}$.

Aunque las tasas específicas de fecundidad en niñas entre los 10 y 14 años no superan siete nacimientos por cada 1000 niñas en esa misma edad en todos los municipios, este indicador se encuentra, en niñas entre los 15 y 19 años, por encima de 48 nacimientos por cada 1000 niñas en esa misma edad. Caloto, Pivijay y Bahía Solano, fueron los municipios que más nacimientos reportaron por cada 1000 niñas entre los 15 y 19 años $^{12}$.

En cuanto a los casos de violencia intrafamiliar contra niñas/os, en el Instituto de Medicina Legal, se encontraron solo registros de Aracataca, Caloto y Dibulla con reportes de 41, 36, y 19 casos de violencia en niñas/os y adolescentes por cada cien mil habitantes, respectivamente ${ }^{10}$. En general, los municipios se encuentran en condiciones de pobreza multidimensional, tienen altas tasas de fecundidad en menores de 19 años y los casos de violencia intrafamiliar contra niñas/os no están siendo reportados en la mayoría de los municipios.

Este estudio hace parte de una intervención social llamada Valiente, (2 018-2 023), financiada por la embajada de Canadá para disminuir las altas tasas de embarazo adolescente y violencia basada en género (VBG) en ocho municipios de Colombia. La investigación de este proyecto tendrá tres momentos, el primero fue desarrollar una línea base que permitió establecerun diagnóstico inicial sobre los conocimientos, percepciones, actitudes y practicas sobre temas de sexualidad, educación integral sobre sexualidad, roles de género y empoderamiento. El segundo momento, será una evaluación intermedia que evaluará los avances de la intervención. Y una evaluación final para medir el impacto de la intervención.

Este estudio, tiene como objetivo describir los conocimientos, actitudes y prácticas en educación sexual integral de 6645 niñas y niños escolarizados en ocho municipios priorizados durante el 2018 en Colombia, teniendo en cuenta el contexto de los municipios y las características de los hogares en los que viven las niñas/os.

\section{Metodología}

\section{Tipo de estudio}

Se realizó un estudio exploratorio descriptivo transversal, de carácter cuantitativo, de los conocimientos, actitudes y prácticas de las niñas/os. Se analizó el contexto en el que se desenvuelven ellos: las características de los municipios; dinámicas del hogar en el que viven; y características de las instituciones educativas donde estudian.

\section{Descripción de la población de estudio}

6645 niñas/os escolarizados que viven en los departamentos del Chocó (Bahía Solano y El Carmen de Atrato); Cauca (Caloto y Miranda); Magdalena (Pivijay y Aracataca); y La Guajira (Dibulla y Uribia). La muestra se seleccionó mediante un muestreo no probabilístico por conveniencia. Los criterios de inclusión y exclusión de la cohorte fueron definidos de acuerdo a las cifras y las necesidades encontradas: niñas/os de instituciones educativas públicas, entre $9 \mathrm{y}$ 11 años, de grados escolares tercero, cuarto y quinto con residencia habitual en el municipio. El criterio de la edad se basó en la teoría del desarrollo cognitivo de Piaget, que explica cómo las niñas/os construyen un modelo mental del mundo y que este momento de la vida es una etapa en la que empiezan a pensar de forma más lógica ${ }^{13}$. Además, existe evidencia en Colombia que la educación integral en sexualidad está llegando solo hasta los 16 años de edad ${ }^{8}$ y no se está implementando durante la vida escolar tal y como lo obliga la Resolución 3353 del Ministerio de Educación Nacional (MEN) $)^{14}$.

\section{Fuente de datos}

Una encuesta de Conocimientos, Actitudes y Prácticas fue diseñada, validada y aplicada, entre octubre de 2018 y marzo de 2019, a 6645 niñas/os entre 9 y 11 años, 
a 5600 hogares y a 50 rectores. Los tres cuestionarios tienen preguntas cerradas lamayoría opción múltiple, con única o multiple respuesta. Los cuestionarios fueron sometidos a prueba piloto y tuvieron ajustes para mejorar la comprensión y fluidez.

El cuestionario de niñas/os tenía 41 preguntas en seis capítulos: I) características y relaciones familiares; II) convivencia escolar y clima educativo; III) educación para la sexualidad; IV) sexualidad, conocimiento y uso de métodos; V) roles de género y poder sexual; $\mathrm{y}$ VI) empoderamiento. El cuestionario de hogares tenía 75 de preguntas en ocho capítulos: I) identificación del hogar; II) condiciones habitacionales del hogar; III) características de las personas del hogar; IV) experiencia de inseguridad alimentaria; V) salud básica y hábitos nutricionales; VI) comportamiento en casa; VII) percepción del entorno; y VIII) roles de género.

El cuestionario de rectores tenía 58 preguntas divididas en 14 capítulos: I) características de la institución educativa (IE); II) ambiente físico de la IE; III) relación entre docentes; IV) relación entre estudiantes; V) proyecto educativo y gestión educativa; VI) patrones de convivencia; VII) ambiente de aprendizaje; VIII) patrones culturales; IX) relación IE - comunidad; $\mathrm{X}$ ) ausentismo y deserción escolar; XI) entorno y convivencia escolar; XII) proyecto de educación para la sexualidad; XIII) embarazo adolescente; y XIV) proyectos transversales de la IE.

El cuestionario de niñas/os y rectores fueron auto diligenciados, el de hogares fue respondido por el jefe del hogar. En el caso del cuestionario de hogares se contrató a personas con experiencia en encuestas poblacionales para que realizarán la encuesta.

\section{Análisis estadístico}

Análisis descriptivo univariado. Se calcularon y analizaron porcentajes para describir las características de hogares, IE y de la población objetivo

\section{Resultados}

Los resultados se presentan así: características de los hogares en la Tabla 1, y los conocimientos, actitudes y prácticas en educación integral en sexualidad de niñas/ os en las Tablas 2, 3 y 4.

Tabla 1. Características de los hogares de las niñas y niños, 2019.

\begin{tabular}{|c|c|c|c|c|c|c|c|c|c|}
\hline & Aracataca & Pivijay & Dibulla & Uribia & Bahía Solano & El Carmen & Caloto & Miranda & Total \\
\hline Categoría & $(\%)$ & $(\%)$ & $(\%)$ & $(\%)$ & $(\%)$ & de Atrato (\%) & $(\%)$ & $(\%)$ & $(\%)$ \\
\hline Jefe de hogar mujer & 56,1 & 31,9 & 36 & 46,8 & 47,4 & 34,3 & 35,6 & 40,5 & 42,9 \\
\hline Acueducto público & 76,1 & 26,8 & 73,8 & 11,6 & 34,4 & 39,5 & 50,1 & 91,7 & 57,3 \\
\hline No tienen sanitario & 7,7 & 7,2 & 6,7 & 11,1 & 12,5 & 0,4 & 0,5 & 0,4 & 6,5 \\
\hline Número de hogares & 1338 & 870 & 1101 & 449 & 529 & 258 & 377 & 678 & 5600 \\
\hline \multicolumn{10}{|l|}{ Pertenencia étnica } \\
\hline Negro/a o afro & 16,1 & 3,3 & 40,6 & 3,6 & 87,2 & 14,2 & 33,8 & 26,8 & 26,8 \\
\hline Indígena & 1,6 & 1,2 & 7,7 & 76,6 & 5,1 & 3,2 & 31,9 & 15,1 & 12 \\
\hline Gitano & 0,5 & 0,4 & 0,2 & 0,5 & 0,2 & 0,1 & 1,2 & 0,3 & 0,4 \\
\hline Ninguna & 78,9 & 86,6 & 49,6 & 17,2 & 7,2 & 77,5 & 28 & 55,6 & 57,3 \\
\hline \multicolumn{10}{|c|}{ Régimen de Seguridad Social en Salud } \\
\hline Contributivo & 24,2 & 5,6 & 16,6 & 19,3 & 5,7 & 38,7 & 36,8 & 41,7 & 20,6 \\
\hline Subsidiado & 70,7 & 90,3 & 74,9 & 67,9 & 84,4 & 52,8 & 58,8 & 52,6 & 72,5 \\
\hline Otro & 1,9 & 1,9 & 1,9 & 6,5 & 4 & 4,9 & 2,5 & 3,3 & 2,8 \\
\hline No está afiliado & 3,2 & 2,2 & 6,6 & 6,3 & 5,9 & 3,6 & 1,8 & 2,4 & 4,1 \\
\hline Total de personas del hogar: & 6490 & 4186 & 4779 & 1960 & 2528 & 1099 & 1265 & 2804 & 25111 \\
\hline \multicolumn{10}{|l|}{ Máximo nivel escolar alcanzado } \\
\hline Ninguno/preescolar & 20,5 & 11,2 & 6,8 & 16,2 & 6,3 & 7,1 & 20,6 & 14,1 & 13,3 \\
\hline Primaria & 62,3 & 63,6 & 64,5 & 54,6 & 62,4 & 66,4 & 65,9 & 59,5 & 62,4 \\
\hline Secundaria & 16,4 & 23,2 & 25,1 & 21,4 & 27,5 & 23,3 & 9,9 & 22,1 & 21,3 \\
\hline Técnico/universitario & 0,8 & 2,1 & 3,6 & 7,9 & 3,8 & 3,1 & 3,6 & 4,3 & 2,1 \\
\hline $\begin{array}{l}\text { Número de personas que ha } \\
\text { asistido a la escuela }\end{array}$ & 5549 & 3503 & 4139 & 1651 & 2141 & 973 & 1095 & 2542 & 21593 \\
\hline
\end{tabular}

Fuente: Elaboración propia basada en Encuesta CAP aplicada a niñas y niños de los ocho municipios priorizados durante el 2018-2019. 
Tabla 2. Características generales de niñas y niños entre 9 y 11 años los ocho municipios, 2019.

\begin{tabular}{|c|c|c|c|c|c|c|c|c|c|c|c|}
\hline Categoría & Aracataca & Pivijay & Dibulla & Uribia & $\begin{array}{c}\text { Bahía } \\
\text { Solano }\end{array}$ & $\begin{array}{c}\text { El } \\
\text { Carmen } \\
\text { de Atrato }\end{array}$ & Caloto & Miranda & Niñas & Niños & Total \\
\hline Vive con los dos padres $(\%)$ & 59,5 & 68,8 & 61,1 & 63,8 & 62,7 & 57,1 & 57,7 & 51,5 & 59,8 & 62,3 & 60,9 \\
\hline Vive solo con la madre (\%) & 12,4 & 10,2 & 16,6 & 16,7 & 20 & 16,6 & 15 & 15,6 & 15,9 & 13,4 & 14,7 \\
\hline Vive solo con el padre (\%) & 0,9 & 1,4 & 1,2 & 1,2 & 0,6 & 1,6 & 1,7 & 1,2 & 1,2 & 1,2 & 1,2 \\
\hline $\begin{array}{l}\text { Dialogo como forma de } \\
\text { corrección }(\%)\end{array}$ & 50,8 & 51,8 & 49,6 & 42,8 & 47,4 & 53,4 & 56,7 & 54,3 & 53,7 & 46,7 & 50,4 \\
\hline $\begin{array}{l}\text { Castigos físicos (con correa, } \\
\text { palo, objetos, encierros...) }(\%)\end{array}$ & 36,9 & 27,7 & 34 & 31,2 & 55,9 & 35,6 & 25,6 & 36,8 & 27,4 & 42,5 & 34,4 \\
\hline $\begin{array}{l}\text { Desacuerdo con: algunas veces } \\
\text { de justifica golpear a las mujeres } \\
(\%)\end{array}$ & 59,7 & 62,8 & 59,8 & 51,3 & 61 & 69,2 & 64,9 & 66,3 & 60,6 & 61,1 & 60,7 \\
\hline $\begin{array}{l}\text { Desacuerdo con: una mujer } \\
\text { debe aguantar la violencia de su } \\
\text { marido para mantener su familia } \\
\text { unida }(\%)\end{array}$ & 54 & 59,2 & 55,6 & 48,4 & 59,2 & 68,8 & 60,2 & 61,2 & 60 & 52,8 & 56,7 \\
\hline $\begin{array}{l}\text { Desacuerdo con: Cuando se } \\
\text { toman decisiones el hombre } \\
\text { tiene la última palabra }(\%)\end{array}$ & 32 & 33,3 & 33 & 27,3 & 37,1 & 39,3 & 40,2 & 35 & 36,8 & 29,4 & 33,3 \\
\hline $\begin{array}{l}\text { Desacuerdo con: el papel más } \\
\text { importante de las mujeres es } \\
\text { cuidar su casa y cocinar }(\%)\end{array}$ & 17,2 & 20,7 & 20,4 & 17,9 & 24,9 & 27,9 & 30,5 & 26,1 & 24 & 19,1 & 21,7 \\
\hline \multirow{4}{*}{$\begin{array}{l}\text { Orientación } \\
\text { sexual }(\%)\end{array}$} & 91,6 & 94,6 & 95,1 & 86,3 & 92,8 & 91,5 & 91,5 & 94 & $\mathrm{NA}^{\mathrm{a}}$ & $\mathrm{NA}^{\mathrm{a}}$ & 92,2 \\
\hline & 3,4 & 1,6 & 1,9 & 5,2 & 3,3 & 4,5 & 3,5 & 2,5 & $\mathrm{NA}^{\mathrm{a}}$ & $\mathrm{NA}^{\mathrm{a}}$ & 3,1 \\
\hline & 2,6 & 1,6 & 1,8 & 4,4 & 1,4 & 2,4 & 1 & 1,2 & $\mathrm{NA}^{\mathrm{a}}$ & $\mathrm{NA}^{\mathrm{a}}$ & 2,2 \\
\hline & 2,4 & 2,1 & 1,2 & 4,2 & 2,5 & 1,6 & 4,1 & 2,3 & $\mathrm{NA}^{\mathrm{a}}$ & $\mathrm{NA}^{\mathrm{a}}$ & 2,5 \\
\hline No sabe que es sexualidad (\%) & 26,1 & 38,1 & 39,3 & 48,8 & 26,2 & 23,9 & 30,7 & 30,3 & 37,7 & 31,1 & 34,7 \\
\hline $\begin{array}{l}\text { Relación de sexualidad con } \\
\text { genitalidad }(\%)\end{array}$ & 50,5 & 43,5 & 47,1 & 36,9 & 58,1 & 60,7 & 55,7 & 54,2 & 45,6 & 51,9 & 48,5 \\
\hline $\begin{array}{l}\text { ¿Han tenido relaciones sexuales? } \\
(\%)\end{array}$ & 0.3 & 1,6 & 1,3 & 0,7 & 6,8 & 2 & 3,9 & 3,7 & 0,4 & 3,9 & 2 \\
\hline $\begin{array}{l}\text { Conocimiento uso de métodos } \\
\text { anticonceptivos }(\%)\end{array}$ & 11,2 & 18,5 & 10,6 & 11,4 & 23,5 & 27,9 & 19,4 & 23,4 & 16,1 & 16,3 & 16,3 \\
\hline Total niñas y niños & 1236 & 1164 & 1042 & 1034 & 485 & 247 & 515 & 922 & 3550 & 3095 & 6645 \\
\hline
\end{tabular}

Fuente: Elaboración propia basada en Encuesta CAP aplicada a niñas y niños de los ocho municipios priorizado durante el 2 018-2 019.

Tabla 3. Fuentes de información sobre sexualidad, niñas y niños entre 9 y 11 años los ocho municipios, 2019.

\begin{tabular}{lccccccccccc}
\multicolumn{1}{c}{ Categoría } & Aracataca & Pivijay & Dibulla & Uribia & $\begin{array}{c}\text { Bahía } \\
\text { Solano }\end{array}$ & $\begin{array}{c}\text { El Carmen } \\
\text { de Atrato }\end{array}$ & Caloto & Miranda & Niñas & Niños & Total \\
\hline $\begin{array}{l}\text { Hablan de sexualidad con } \\
\text { mamá (\%) }\end{array}$ & 59,5 & 53,4 & 52,2 & 41,4 & 48,6 & 52,1 & 56,9 & 55,2 & 62,4 & 43,5 & 53,2 \\
$\begin{array}{l}\text { Hablan de sexualidad con } \\
\text { papá (\%) }\end{array}$ & 35,9 & 34,5 & 26,6 & 23,4 & 26,5 & 23,4 & 29,1 & 24 & 23,9 & 34,7 & 29,2 \\
$\begin{array}{l}\text { No habla de sexualidad con } \\
\text { nadie (\%) }\end{array}$ & 18,7 & 24,7 & 24,7 & 35,3 & 17,6 & 7,4 & 25,8 & 27,8 & 23,7 & 24,2 & 24 \\
$\begin{array}{l}\text { Colegio: } \\
\text { información en sexualidad } \\
\text { (\%) }\end{array}$ & 15 & 15,7 & 12,8 & 15,3 & 11,7 & 16 & 17,4 & 22,4 & 16,2 & 15,5 & 15,9 \\
\begin{tabular}{l} 
Total niñas y niños \\
\hline
\end{tabular} & 914 & 721 & 632 & 529 & 358 & 188 & 357 & 643 & 2213 & 2102 & 4342 \\
\hline
\end{tabular}

Fuente: Elaboración propia basada en Encuesta CAP aplicada a niñas y niños de los ocho municipios priorizado durante el 2018-2019. 
Tabla 4. Relaciones y sexualidad, niñas y niños entre 9 y 11 años los ocho municipios, 2019.

\begin{tabular}{|c|c|c|c|c|c|c|c|c|c|c|c|}
\hline Categoría & Aracataca & Pivijay & Dibulla & Uribia & $\begin{array}{l}\text { Bahía } \\
\text { Solano }\end{array}$ & $\begin{array}{c}\text { El } \\
\text { Carmen } \\
\text { de Atrato }\end{array}$ & Caloto & Miranda & Niñas & Niños & Total \\
\hline $\begin{array}{l}\text { Primera pareja sexual: } \\
\text { novia/o (\%) }\end{array}$ & 25 & 52,6 & 28,6 & 42,9 & 63,6 & 60 & 70 & 44,1 & 26,7 & 55,8 & 52,2 \\
\hline $\begin{array}{l}\text { Primera pareja sexual: } \\
\text { amiga/o (\%) }\end{array}$ & 50 & 26,3 & 21,4 & 14,3 & 12,1 & 20 & 25 & 32,4 & 20 & 24,2 & 23,5 \\
\hline $\begin{array}{l}\text { Primera pareja sexual: } \\
\text { prima/o (\%) }\end{array}$ & 25 & 0 & 7,1 & 28,6 & 12,1 & 20 & 0 & 8,8 & 20 & 7,5 & 8,8 \\
\hline $\begin{array}{l}\text { Edad persona con la que tuvo } \\
\text { relación sexual: misma edad } \\
(\%)\end{array}$ & 66,7 & 64,7 & 54,5 & 80 & 82,1 & 33,3 & 72,2 & 60,6 & 50 & 69,5 & 67,8 \\
\hline $\begin{array}{l}\text { Edad persona con la que tuvo } \\
\text { relación sexual: } 1 \text { a } 5 \text { años } \\
\text { mayor (\%) }\end{array}$ & 33,3 & 11,8 & 27,3 & 20 & 7,1 & 33,3 & 16,7 & 18,2 & 41,7 & 13,3 & 16,1 \\
\hline Total niñas y niños & 4 & 19 & 14 & 7 & 33 & 5 & 20 & 34 & 15 & 120 & 136 \\
\hline
\end{tabular}

Fuente: Elaboración propia basada en Encuesta CAP aplicada a niñas y niños de los ocho municipios priorizados durante el 2018-2019.

\section{Hogares}

En los 5600 hogares de las niñas/os, la distribución entre hombres y mujeres entre los 20 y los 39 años, predominó el número de mujeres sobre el número de hombres en el hogar. El 42,9\% del total de hogares encuestados tenía jefatura femenina. Como se puede observar en la Tabla 1, en Aracataca (56,1\%) y Bahía Solano (47,4\%) se encontró el porcentaje más alto. En términos generales, el $26,8 \%$ de las personas que conformaban los hogares se identificó como negro o afrodescendiente, el $12 \%$ como indígena y menos del $1 \%$ como gitano. El 72,5\% de las personas del hogar está afiliado al Sistema de Salud den régimen subsidiado. El máximo nivel escolar alcanzado por el $62,4 \%$ de los hogares fue primaria, seguido por secundaria $(21,3 \%)$. Entre las mujeres de 6 a 24 años que no asisten al colegio, el 13,3\% dejaron de estudiar porque no querían o porque quedaron embarazadas. Entre los hombres, del $36 \%$ que no quiso estudiar, el 12,6\% necesitaba ganar dinero, y el 10,6\% no podía pagar los costos de estudio. Al momento de la encuesta se encontraron seis niñas embarazadas entre 10 y 14 años en los municipios de Dibulla y Miranda, tres en cada municipio. Entre los 15 y 19 años, se encontraron adolescentes embarazadas en cuatro de los ocho municipios: 25 en Bahía Solano; 52 en Aracataca; 36 en Dibulla; y 24 en Pivijay.

Muchos de los hogares en los que viven las niñas/os presentaron malas condiciones habitacionales. Más del $20 \%$ de los hogares no cuenta con acceso al servicio público de acueducto; en Miranda, Caloto y Bahía Solano la cobertura es superior al $90 \%$, mientras que, en Uribia menos del $50 \%$ de los hogares cuenta con este servicio. El 6,5\% de los hogares no tiene sanitario, siendo Bahía Solano (12,5\%) y Uribia $(11,1 \%)$ los municipios con más hogares en esta situación.

Cuando se evaluó la seguridad alimentaria de los hogares, es decir, la preocupación de los hogares porque los alimentos se acabarán, la reducción de alimentos disponibles, la calidad de la alimentación y la situación de hambre por falta de dinero, se encontró que más del $20 \%$ presentaron inseguridad alimentaria severa, el $16 \%$ inseguridad moderada, el $26 \%$ leve y el $35 \%$ tienen seguridad alimentaria.

\section{Instituciones educativas}

De acuerdo a la información de los rectores, entre las principales causas de abandono escolar se encontraron: cambio de domicilio de los hogares; extra-edad en los grados escolares; poca importancia, por parte de los padres, con la educación de sus hijos o no los motivan a estudiar; dificultades académicas; y poco gusto por el estudio en las niñas/os.

Bajo rendimiento escolar, embarazos no deseados, ausentismo escolar, conflictos o peleas, robo de elementos y abuso sexual, son los problemas que más refieren los rectores que se presentan en las IE. Los motivos porque se presentan estos problemas son el juego brusco, abuso de confianza por apodos o sobrenombres, chismes o malos entendidos, falta de apoyo de los padres o acudientes y la ausencia de valores en los estudiantes. 
E1 $72 \%$ de las IE refirió tener un Proyecto de Educación para la Sexualidad (PES) y de estas, el 88,9\% informó seguir los lineamientos del del MEN. Las principales causas para no seguir los lineamientos fueron: no eran aplicables a la comunidad; no tenían continuidad en el proyecto y realizan el proyecto de acuerdo a sus propios problemas.

El $44 \%$ de las IE cuenta con un registro de niñas embarazadas que desertaron del sistema educativo. Con relación a las actividades que desarrolla la IE encaminadas a disminuir el embarazo adolescente y la violencia intrafamiliar, todas refirieron por lo menos realizar una actividad. Entre las principales actividades se encuentran: charlas, talleres, articulaciones con el sistema de salud y escuela de padres.

Los principales programas que realizan los estudiantes, y que les permiten fortalecer el crecimiento integral, así como también permite la disminución de las violencias de género y el embarazo adolescente, son: educación ambiental; actividades de recreación y deporte; programas artísticos; aprovechamiento del tiempo libre y hábitos de vida saludable.

\section{Niñas y niños}

Cuando se les preguntó sobre el sexo de la persona por la cual sienten atracción física, emocional o erótica, más del $90 \%$ respondieron que, por personas del sexo opuesto, el $3 \%$ por personas del mismo sexo y alrededor del $2 \%$ por ambos sexos. Uribia, presentó los porcentajes más altos en las últimas dos categorías mencionadas. En las Tablas 2, 3 y 4, se detallan los resultados de las niñas/os. Más del $50 \%$ viven con el papá y la mamá, alrededor del $23 \%$ solo vive con la mamá y el 2,8\% solo con el papá. En cuanto a la manera como los corrigen, el dialogo $(60 \%)$ es la principal forma de corrección y más del $30 \%$ respondió que los castigan físicamente, siendo los niños quienes reciben más castigos físicos ( $42 \%$ frente a $27 \%$ ).

La mamá $(53,2 \%)$ es el principal referente para hablar de sexualidad, seguido del papá $(29,2 \%)$. El $24 \%$ respondieron no hablar con nadie sobre este tema. Sin embargo, las niñas hablan más con las mamás que los niños, quienes tienden a conversar sobre estos temas con los amigos y con los papás. Solo el $15,9 \%$ de las niñas/os refieren que las instituciones educativas son una fuente de información sobre sexualidad. Hay desconocimiento del concepto sexualidad, particularmente en Dibulla y Uribia. Hay una común relación de este término con la genitalidad ("tener relaciones sexuales", "hacer el amor" y "tener sexo para tener hijos/as"), sobre todo visible en Bahía Solano, El Carmen de Atrato, Miranda y Caloto.

Cuando se les preguntó a las niñas/os si recibieron información en los últimos meses sobre temas de autoconocimiento, relaciones interpersonales, decisiones sexuales, decisiones reproductivas, auto cuidado, mutuo cuidado o socio cuidado, el 51,8\% respondieron que se les ha hablado sobre cambios que presentan en la adolescencia. Sin embargo, cuando se les preguntó sobre temas como la orientación sexual, la igualdad de género, uso de métodos anticonceptivos, VBG, entre otros, menos del $40 \%$ respondieron haber recibido alguna información al respecto. Cuando se realizó el análisis discriminado por sexo biológico, se encontró que los niños recibieron más información que las niñas sobre decisiones reproductivas, como el uso de métodos anticonceptivos, el placer y el erotismo, mientras que las niñas recibieron más información sobre temas relacionados con el abuso sexual, el acoso y la discriminación.

Se preguntó el nivel de acuerdo o desacuerdo a ciertos enunciados, para identificar aspectos relacionados con la violencia contra las mujeres. Hubo un nivel de acuerdo importante respecto a que la mujer no debe aguantar este tipo de violencia (más del $50 \%$ ); sin embargo, en los enunciados que hacían referencias más sutiles a la desigualdad y la violencia dentro de la pareja, como "cuando se tienen que tomar las decisiones en la casa, los hombres tienen la última palabra" y "el papel más importante de las mujeres es cuidar su casa y cocinar para su familia", se presentan bajos niveles de desacuerdo (35\%).

133 niñas/os refieren ya haber tenido relaciones sexuales y hay una diferencia de 3,5 puntos porcentuales al realizar el análisis discriminado por sexo, donde los niños son quienes más han tenido relaciones sexuales. Las niñas/ os de Bahía Solano, Miranda y Caloto presentaron el mayor porcentaje de respuesta afirmativa. Las niñas/os tuvieron su primera relación sexual principalmente con su novio/a, amigo/a o un primo/a. El $68 \%$ tuvieron su primera relación con una persona de la misma edad, el $16 \%$ con una persona uno o cinco años mayor. Menos del $20 \%$ conocen algún método anticonceptivo, y de todos los métodos, el condón es el más conocido, particularmente más por los niños. 


\section{Discusión}

En los municipios analizados se encuentran altos niveles de pobreza, una alta densidad poblacional en zonas rurales y altas tasas de fecundidad en adolescentes. En las IE se encontró alta movilidad de niñas/os por cambio de domicilio, bajo rendimiento académico, embarazos no deseados; actitudes violentas entre compañeros y falta de apoyo de los padres o cuidadores. Mientras tanto, en la encuesta a niñas/os, se encontraron altos porcentajes de desacuerdo de la violencia directa contra la mujer y validación de formas sutiles de violencia; alto desconocimiento del concepto sexualidad y quienes creen conocer el termino, lo dialogan principalmente con su madre y un porcentaje importante refieren no hablarlo con nadie. Además, las niñas/os están iniciando relaciones sexuales a edades tempranas.

De acuerdo al reporte sobre Pobreza, Salud Sexual y Derechos Humanos del Fondo de Población de las Naciones Unidas, mejorar la calidad de los indicadores de SSR podría maximizar su impacto en la pobreza, equidad y derechos humanos ${ }^{15}$. Los ocho municipios priorizados en este estudio tienen cifras elevadas de pobreza y altas tasas de fecundidad en niñas menores de 19 años, lo que hace un llamado a trabajar fuertemente por mejorar los indicadores de SSR de todos los municipios y con ello, mejorar su desarrollo integral. Para que esto suceda, las actividades de promoción de los derechos sexuales y reproductivos se deben implementar teniendo en cuenta la evidencia científica ${ }^{16}$.

En este estudio se presentaron altos niveles de hogares monoparentales en donde la jefe del hogar es mujer. Como lo documenta la ENDS 2015, hay un incremento de los hogares unipersonales, así como una disminución de las familias nucleares biparentales, extensas y compuestas y el aumento de las nucleares monoparentales y de las parejas sin hijos ${ }^{8}$.

Una de las principales razones de no asistencia al colegio de las mujeres de 6 a 24 años del hogar fue haber quedado en embarazo, por el contrario, en los hombres, que su pareja haya quedado en embarazo, no estuvo entre sus primeras opciones de no asistencia escolar. La edad y asistir a actividades escolares determinan en gran medida que ocurra o no el embarazo en la adolescencia, siendo la deserción escolar uno de los factores de riesgo ${ }^{8}$. La salud materna representa una carga dominante en las mujeres ${ }^{15}$. La evidencia demuestra la relación entre las altas tasas de fertilidad en adolescentes y los bajos niveles educativos; incluso las niñas que asisten a la escuela con frecuencia se ven forzadas a abandonarla durante el embarazo, lo que pone en riesgo sus posibilidades económicas futuras ${ }^{4}$.

Aunque las IE tienen un potencial importante para la transformación social, también deben ser entendidas como espacios de reproducción de los valores existentes que a menudo entran en tensión con proyectos como la educación integral para la sexualidad y las transformaciones en los roles tradicionales de género $^{17}$. Está reproducción de valores y enfoque en proyectos sobre educación sexual deben ser iniciados y potenciados en cada una de las IE donde se encontraron altos niveles de violencia entre los estudiantes, poco interés por el estudio, desinterés por padres de familia, además de las altas tasas de embarazo adolescente.

El dialogo es la principal forma de corrección para niños, pero más del $30 \%$ respondió que los castigan físicamente (con correa, palo, otro objeto, les dan palmadas, pellizcos, los encierran o los aíslan). Existe evidencia que demuestra que cuanto más golpeados son las niñas/os, más enojo reportan en la edad adulta, más golpean a sus propios hijos/as cuando llegan a ser padres y más tienden a golpear a sus parejas. Así se produce la transmisión intergeneracional de la violencia ${ }^{18}$.

En las niñas/os hubo un fuerte desconocimiento del término sexualidad y una relación por parte de ellas/os con el concepto de genitalidad. Con relación al acceso a información sobre la sexualidad, la ENDS 2015 reveló que, en promedio las mujeres de 13 a 49 años tenían 16 años la primera vez que accedieron a información sobre sexualidad ${ }^{8}$. La diferencia de oportunidad de acceso a la información es de siete años entre las mujeres con menor nivel educativo, quienes recibieron información sobre sexualidad por primera vez a los 22 años, y las mujeres con educación superior que la recibieron por primera vez a los 15 años.

Entre las niñas/os entre 9 y 11 años, más del $15 \%$ refieren haber tenido su primera relación sexual con una pareja uno a cinco años mayor y fueron las niñas quienes tuvieron más diferencia de edad con la pareja. Según la ENDS 2015 el 17\% de las mujeres de 13 a 19 años tuvo su primera relación sexual antes de los 14 , en el $20 \%$ de estos casos la relación fue con una pareja 6 o 9 años mayor y en el 19,6\% de los casos con una pareja 10 años mayor. Las mayores diferencias de edad alertan sobre la asimetría de poder que vulnera la capacidad de decisión, de acceso a la información y de comprensión de las consecuencias de las propias decisiones ${ }^{8}$. 
Los resultados reflejan los conocimientos y actitudes de las niñas, niños y adolescentes (NNA) frente a los asuntos relacionados con sexualidad, estereotipos de género, violencia contra la mujer, la necesidad insatisfecha de acceder a información de calidad y uso de métodos anticonceptivos. La información disponible es escasa y la poca información que tienen sobre estos temas es errada. Esto es un signo de alarma, ya que se ha demostrado que la falta de acceso e información sobre los servicios de SSR, contribuye a que existan embarazos no deseados y a que las infecciones de transmisión sexual sean una de las principales causas de muerte a nivel global en las niñas entre 10 y 19 años $^{4}$. Es importante definir estrategias de comunicación en cada uno de los municipios, que permitan construir mejores actitudes en favor de estos temas y se deben definir captando a los NNA como sujetos de derechos y que se dejen atrás, las construcciones idílicas de vulnerabilidad y buscando siempre la garantía y goce efectivo de esto ${ }^{19}$.

En conclusión, en todos los municipios a intervenir es necesario profundizar el significado de la sexualidad y la VBG con el fin de lograr promover y defender un proyecto de Educación Integral para la Sexualidad que se entienda como una intervención que, desarrollada apropiadamente, influye positivamente en la toma de decisiones de los adolescentes y por ende en sus proyectos de vida.

Entre las limitaciones de este estudio se encuentra que los resultados obtenidos no son representativos para los municipios, por lo cual, impide la realización de inferencias estadísticas. Una segunda limitación es que la encuesta de NNA fue autodiligenciada, lo cual, pudo generar sesgos en entendimiento de algunas preguntas.

\section{Agradecimientos}

Esta investigación se realizó gracias a la participación de las niñas y niños que se tomaron el tiempo de responder a cada una de las preguntas de la encuesta y a las personas que realizaron el trabajo de campo en cada uno de los municipios. Apoyos recibidos: Este estudio hace parte de una intervención social financiada por la embajada de Canadá y la Asociación Profamilia en Colombia.

\section{Consideraciones éticas}

Esta investigación fue aprobada por el Comité de ética en investigación de Profamilia el 17 de octubre de 2018. Se contó con el asentimiento escrito de los menores de edad y el consentimiento escrito de las personas encargadas de responder el cuestionario hogares y así mismo el de los rectores/coordinadores. En todos los cuestionarios se explicó el carácter voluntario de su participación y la posibilidad de no contestar alguna pregunta en caso de no querer hacerlo, o de dar por terminada la encuesta en cualquier momento. Todos los cuestionarios se realizaron en condiciones de privacidad y no se dio ninguna compensación económica por participar.

\section{Conflicto de intereses}

Los autores declaran no tener conflicto de intereses.

\section{Referencias}

1. PNUD. Objetivos de Desarrollo Sostenible PNUD. Programa de las Naciones Unidas para el Desarrollo. 2018.

2. Barrero-Barrero D, Baquero-Valdés F. Sustainable development goals: A post-modern social contract for justice, development, and security. Rev Cient Gen Jose Maria Cordova. 2020; 18(29): 113-137.

3. OMS. El embarazo en la adolescencia. Organización Mundial de la Salud. 2018.

4. Godt S, Agyepong I, Flores W, Sen G. Una vida sana para mujeres y niños vulnerables. Centro Internacional de Investigaciones para el Desarrollo (IDRC); 2017. $125 \mathrm{p}$.

5. Murad-Rivera R, Rivillas-García JC, Victoria V, Forero-Martínez LJ. Determinantes del embarazo en adolescentes en Colombia: Explicando las causas de las causas. Asoc Profamilia. 2018.

6. UNESCO. Por qué es importante la educación integral en sexualidad. 15/02/2018. 2018. p. PG1. https://es.unesco.org/news/que-es-importanteeducacion-integral-sexualidad

7. Ministerio de Salud y Protección Social -MSPS. Encuesta Nacional de Demografía y Salud 2015 Tomo 1: Componente Demográfico. Profamilia. 2015.

8. Profamilia - Ministerio de Salud y Protección Social. Encuesta Nacional de Demografía y Salud 2015 Tomo II: Componente de salud sexual y salud reproductiva. Minsalud. 2017.

9. Ministerio de Salud y Protección Social. Análisis de la línea base del Observatorio de Embarazo Adolescente en Colombia. 2013.

10. Instituto Nacional de Medicina Legal y Ciencias Forenses. Forensis 2017 Datos para la vida. 2018.

11. Departamento Nacional de Estadística DANE. Pobreza Monetaria; 2019. 
12. DANE. Estadísticas Vitales de Nacimientos y Defunciones; 2016.

13. Piaget J. Part I: Cognitive development in children: Piaget development and learning. J Res Sci Teach. 2003; 40: nS1: S8-S18.

14. Ministerio de Educación Nacional (MINEDUCACIÓN). RESOLUCIÓN 3353 DE 1993. Revista Legislación Económica Nº: 982.1993.

15. Fondo de Población de las Naciones Unidas (UNFPA). Pobreza, salud sexual y Derechos Humanos. México DF; 2005.

16. Vargas-Trujillo, Cortés, Gallego, Maldonado, Ibarra Ávila, M P, et al. Educación sexual de mujeres colombianas en la juventud: un análisis desde el enfoque basado en los derechos humano. Serie de Estudios aprofundidad ENDS 1990-2010. 2013; 7.

17. Ohsako TE. Violence at School: Global Issues and Interventions. Studies in Comparative Education. 1997; $127 \mathrm{p}$.

18. Sauceda-García JM, Olivo-Gutiérrez NA, Gutiérrez J, Maldonado-Durán JM. El castigo físico en la crianza de los hijos. Un estudio comparativo. Bol Med Hosp Infant Mex. 2006; 63: 382-388.

19. Cely P. Análisis de los niños, niñas y adolescentes como sujetos de derechos. Salud Soc. 2015; 2(1): 42-47. 